Document downloaded from:

http://hdl.handle.net/10251/52264

This paper must be cited as:

Liu, X.; Zhang, M.; Benítez López, J. (2014). Further results on the reverse order law for the group inverse in rings. Applied Mathematics and Computation. 229:316-326. doi:10.1016/j.amc.2013.12.030.

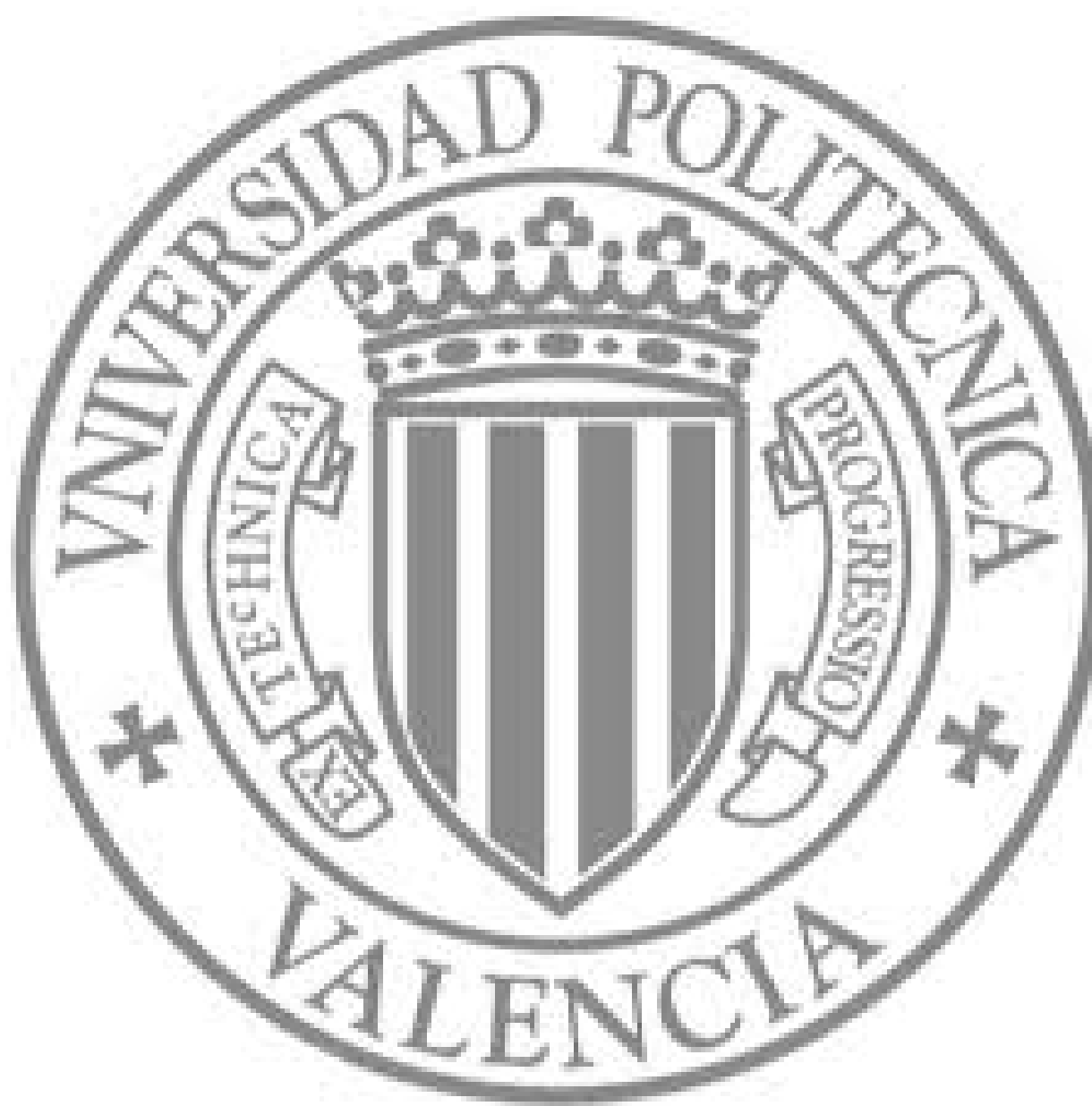

The final publication is available at

http://dx.doi.org/10.1016/j.amc.2013.12.030

Copyright Elsevier 


\title{
FURTHER RESULTS ON THE REVERSE ORDER LAW FOR THE GROUP INVERSE IN RINGS *
}

\author{
XIAOJI LIU ${ }^{\dagger}$, MIAO ZHANG ${ }^{\ddagger}$, AND JULIO BENíTEZ§
}

\begin{abstract}
In this paper, we use the Drazin inverse to derive some new equivalences of the reverse order law for the group inverse in unitary rings. Moreover, if the ring has an involution, we present more equivalences when both involved elements are EP.
\end{abstract}

Keywords: Group inverse; Drazin inverse; Moore-Penrose inverse; reverse order law

AMS-Subject Classification: 15A09, 16W10.

1. Introduction. Let $\mathcal{R}$ be a unitary ring whose unity is $\mathbb{1}$. For $a, b \in \mathcal{R}$, the commutator of $a, b$ is defined as $[a, b]=a b-b a$. Let $a \in \mathcal{R}$. It can be easily proved that the set of $x \in \mathcal{R}$ satisfying the following conditions

$$
a x a=a, \quad x a x=x, \quad a x=x a
$$

is either empty or a singleton. When there exists such $x$, then $a$ is said to be group invertible and $x$ is denoted by $a^{\#}$. A useful fact about group inverses is the following: If $a$ is a group invertible element of a unitary ring $\mathcal{R}$, then $a^{\#}$ double commutes with $a$, that is, if $z \in \mathcal{R}$ satisfies $[a, z]=0$, then $\left[a^{\#}, z\right]=0$ (see e.g. [6, Lemma 1.4.5]). We shall denote by $\mathcal{R}^{\#}$ the subset of $\mathcal{R}$ consisting of group invertible elements and by $\mathcal{R}^{-1}$ the set of standard invertible elements. If $a \in \mathcal{R}^{\#}$, the spectral idempotent of $a$ is defined as $a^{\pi}=\mathbb{1}-a a^{\#}$. Also, the following result on group inverses will be used. For the proof, the interested reader can consult [15, Proposition 8.22].

THEOREM 1.1. Let a be an element of a unitary ring $\mathcal{R}$. Then a is group invertible if and only if exist $x, y \in \mathcal{R}$ such that $a^{2} x=a$ and $y a^{2}=a$. In this case, one has $a^{\#}=$ yax.

With each element $a$ of a unitary ring $\mathcal{R}$ we associate two right ideals:

$$
a \mathcal{R}=\{a x: x \in \mathcal{R}\}, \quad a^{\circ}=\{x \in \mathcal{R}: a x=0\} .
$$

*Received by the editors on Month x, 200x. Accepted for publication on Month y, 200y Handling Editor: .

${ }^{\dagger}$ College of Science, Guangxi University for Nationalities, Nanning 530006, P.R. China, xiaojiliu@yahoo.com.cn

${ }^{\ddagger}$ College of Science, Guangxi University for Nationalities, Nanning 530006, P.R. China, zhangmiao198658@163.com

§Instituto de Matemática Multidisciplinar, Universidad Politécnica de Valencia, Valencia 46022, Spain,jbenitez@mat.upv.es 
Let $a, b$ be elements of a unitary ring $\mathcal{R}$. The element $b$ is a Drazin inverse of $a$ if

$$
a b=b a, \quad b=a b^{2}, \quad a^{k}=a^{k+1} b
$$

for some nonnegative integer $k$. It can be proved (see [7, Theorem 1]) that such $b$ is unique and it is customarily denoted $a^{d}$. The least nonnegative integer $k$ for which these equalities hold is the Drazin index $i(a)$ of $a$. In [7, Theorem 4] it was proved that an element $a \in \mathcal{R}$ is Drazin invertible if and only if there are nonnegative integers $p, q$ and $u, v \in \mathcal{R}$ such that $a^{p+1} u=a^{p}$ and $v a^{q+1}=a^{q}$. The smallest value of $p$ for which $\left\{u \in \mathcal{R}: a^{p+1} u=a^{p}\right\} \neq \emptyset$ is called the left index of $a$, denoted by $l(a)$. In a similar way the right index of $a$ is defined, and is denoted by $r(a)$. In a remark following [7, Theorem 4] it was shown that in case that $a$ is Drazin invertible, then $i(a)=l(a)=r(a)$.

An involution in a ring $\mathcal{R}$ is a map $a \mapsto a^{*}$ such that for any $a, b \in \mathcal{R}$,

$$
\left(a^{*}\right)^{*}=a, \quad(a+b)^{*}=a^{*}+b^{*}, \quad(a b)^{*}=b^{*} a^{*} .
$$

If the ring $\mathcal{R}$ has an involution, then we can define another class of generalized inverse. An element $a \in \mathcal{R}$ is Moore-Penrose invertible if there exists $x \in \mathcal{R}$ such that

$$
a x a=a, \quad x a x=x, \quad(a x)^{*}=a x, \quad(x a)^{*}=x a .
$$

Such $x$, when exists, is unique and is denoted by $a^{\dagger}$.

An element $a \in \mathcal{R}$, where $\mathcal{R}$ is a ring with involution, is said to be self-adjoint when $a=a^{*}$. We say that $a$ is $E P$ when $a$ is Moore-Penrose invertible and $a a^{\dagger}=a^{\dagger} a$. Observe that when $a$ is EP, then $a$ is group invertible and $a^{\dagger}=a^{\#}$, and that any self-adjoint element, Moore-Penrose invertible or group invertible, is EP.

The reverse order law for generalized inverses plays an important role in many areas including singular matrix problem, ill-posed problems, optimization, and statistics (see e.g. $[2,8,16,17,18,19,20]$. These problems have attracted considerable attention since the middle 1960s and many interesting results have been obtained. T.N.E. Greville [9] proved that $(A B)^{\dagger}=B^{\dagger} A^{\dagger}$ if and only if $R\left(A^{*} A B\right) \subseteq R(B)$ and $R\left(B B^{*} A^{*}\right) \subseteq R\left(A^{*}\right)$, for complex matrices $A$ and $B$, where $R(\cdot)$ denotes the column space. This result was extended to linear bounded operators on Hilbert spaces in [10]. Another characterization of the reverse order law for the Moore-Penrose inverse is due to Arghiriade [1]: For complex matrices $A, B$ such that $A B$ exists, $(A B)^{\dagger}=B^{\dagger} A^{\dagger}$ if and only if $A^{*} A B B^{*}$ is EP. The interested reader can consult [2, Section 4.4] for the proof of the original results of Greville and Arghiriade. Later, the reverse order law for the Moore-Penrose inverse was considered in rings with involution (see [11]). C.Y. Deng [4] presented some equivalent conditions concerning the reverse order law $(A B)^{\#}=B^{\#} A^{\#}$ for group invertible operators $A, B$ on a Hilbert space. N.Č. Dinčić 
and D.S. Djordjević [5] gave new equivalences of the reverse order law for the MoorePenrose inverse for operators on Hilbert spaces. D. Mosić and D.S. Djordjević [13] investigated some necessary and sufficient conditions for the reverse order law for the group inverse in rings. The hybrid reverse order law $(a b)^{\#}=b^{\dagger} a^{\dagger}$ in rings was studied in [14].

In this paper, we give new equivalent conditions of the reverse order law for the group inverse in unitary rings. Later, we state some new results related to the reverse order law for the group inverse when both involved elements are EP.

The word idempotent will be reserved for an element $p$ of a unitary ring $\mathcal{R}$ such that $p^{2}=p$. Also, we will write $\bar{p}=\mathbb{1}-p$. If in addition $\mathcal{R}$ has an involution, then we will say that an element $p$ is a projection when $p=p^{2}=p^{*}$.

2. Preliminary results. If $\mathcal{R}$ is a unitary ring and $p \in \mathcal{R}$ is an idempotent, then every $x \in \mathcal{R}$ has the following matrix representation

$$
x=\left[\begin{array}{ll}
p x p & p x \bar{p} \\
\bar{p} x p & \bar{p} x \bar{p}
\end{array}\right]_{p}
$$

If, in addition, $\mathcal{R}$ has an involution and $p$ is a projection, then the above matrix representation preserves this involution. More precisely, if $x \in \mathcal{R}$ is represented as

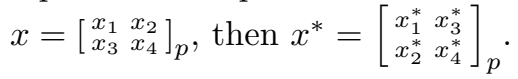

If $a$ is an element of a ring $\mathcal{R}$, then

$a \in \mathcal{R}^{\#} \Longleftrightarrow$ there is an idempotent $p \in \mathcal{R}$ such that $a+p \in \mathcal{R}^{-1}$ and $a p=p a=0$.

Such a $p$, when it exists, is unique (see [15, Proposition 8.24]). This unique idempotent $p$ is the spectral idempotent of $a$-recall that the spectral idempotent of $a$ is customarily written by $a^{\pi}$ and $a^{\pi}=\mathbb{1}-a a^{\#}$ holds. Hence, if $a \in \mathcal{R}^{\#}$, we can represent

$$
a=\left[\begin{array}{ll}
a & 0 \\
0 & 0
\end{array}\right]_{a a^{\#}}, \quad a^{\#}=\left[\begin{array}{cc}
a^{\#} & 0 \\
0 & 0
\end{array}\right]_{a a^{\#}}, \quad a^{\pi}=\left[\begin{array}{cc}
0 & 0 \\
0 & a^{\pi}
\end{array}\right]_{a a^{\#}} .
$$

When (2.1) is applied to a group invertible matrix $A \in \mathbb{C}^{n \times n}$, by writing the idempotent $A^{\pi}$ as $U\left(0 \oplus I_{k}\right) U^{-1}$, where $U \in \mathbb{C}^{n \times n}$ is nonsingular and $I_{k}$ denotes the identity matrix of order $k$ (see e.g. [21, Theorem 5.1]), one easily gets the existence of a nonsingular matrix $B \in \mathbb{C}^{(n-k) \times(n-k)}$ such that $A=U(B \oplus 0) U^{-1}$. Obviously, we have also $A^{\#}=U\left(B^{-1} \oplus 0\right) U^{-1}$.

Lemma 2.1. Let $\mathcal{R}$ be a unitary ring and $m \in \mathcal{R}$. If $p \in \mathcal{R}$ is an idempotent,

$$
m=\left[\begin{array}{ll}
a & b \\
0 & c
\end{array}\right]_{p}
$$


then

(i) If $m$ is group invertible and $c$ is Drazin invertible, then a, $c$ are group invertible and $a^{\pi} b c^{\pi}=0$.

(ii) If $a, c$ are group invertible and $a^{\pi} b c^{\pi}=0$, then $m$ is group invertible and

$$
m^{\#}=\left[\begin{array}{cc}
a^{\#} & \left(a^{\#}\right)^{2} b c^{\pi}+a^{\pi} b\left(c^{\#}\right)^{2}-a^{\#} b c^{\#} \\
0 & c^{\#}
\end{array}\right]_{p} .
$$

Proof. (i) Assume that $m$ is group invertible and $c$ is Drazin invertible. Let us write

$$
m^{\#}=\left[\begin{array}{cc}
u & v \\
w & z
\end{array}\right]_{p}
$$

From $m^{2} m^{\#}=m$ we get

$$
c^{2} w=0
$$

and

$$
c^{2} z=c
$$

Equality (2.6) implies $l(c) \leq 1$. Since $c$ is Drazin invertible we get $i(c)=l(c) \leq 1$. Thus, $c$ is group invertible. By premultiplying by $c^{\#}$ the equality $(2.5)$ we get

$$
c w=0 .
$$

By using $m\left(m^{\#}\right)^{2}=m^{\#}$ and (2.7) we get $c z w=w$. From (2.6) and $c \in \mathcal{R}^{\#}$ we get $c z=c^{\#} c$. Hence (2.7) leads to $w=c z w=c^{\#} c w=0$. By looking at the "north-west" and "south-east" blocks of $m m^{\#} m=m, m^{\#} m m^{\#}=m^{\#}$, and $m m^{\#}=m^{\#} m$ when (2.3), (2.4), and $w=0$ are used, one gets the group invertibility of $a, a^{\#}=u$, and $c^{\#}=z$.

From $m^{2} m^{\#}=m$ we have

$$
a^{2} v+a b c^{\#}+b c c^{\#}=b,
$$

Premultiplication of $(2.8)$ by $\left(a^{\#}\right)^{2}$ yields $a a^{\#} v+a^{\#} b c^{\#}+\left(a^{\#}\right)^{2} b c c^{\#}=\left(a^{\#}\right)^{2} b$, or equivalently

$$
a a^{\#} v=\left(a^{\#}\right)^{2} b c^{\pi}-a^{\#} b c^{\#} .
$$

From $m^{\#} m^{2}=m$ we get $a^{\#} a b+a^{\#} b c+v c^{2}=b$. Similarly as before, postmultiplication of this last equality by $\left(c^{\#}\right)^{2}$ gives

$$
v c c^{\#}=a^{\pi} b\left(c^{\#}\right)^{2}-a^{\#} b c^{\#} .
$$


Finally, from $m^{\#} m m^{\#}=m^{\#},(2.9)$, and (2.10) one obtains

$$
v=a a^{\#} v+a^{\#} b c^{\#}+v c c^{\#}=\left(a^{\#}\right)^{2} b c^{\pi}+a^{\pi} b\left(c^{\#}\right)^{2}-a^{\#} b c^{\#} .
$$

Insertion of this expression of $v$ in (2.8) and a further simplification lead to $a^{\pi} b c^{\pi}=0$.

(ii) follows by verifying that the expression for $m^{\#}$ obeys (1.1), which has a unique solution, namely the group inverse of $m$.

Remark 2.2. Evidently, Lemma 2.1 is valid for complex matrices $M=\left[\begin{array}{ll}A & B \\ 0 & C\end{array}\right]$ for $A \in \mathbb{C}^{n \times n}$ and $C \in \mathbb{C}^{m \times m}$. This case was obtained in [12]. In this setting, the assumption of the Drazin invertibility of $C$ can be removed because any square complex matrix has a unique Drazin inverse (see e.g., [2, Section 4.6]).

REMARK 2.3. A special case of Lemma 2.1 is the following: Let $\mathcal{R}$ be a unitary ring and $m \in \mathcal{R}$. If $p \in \mathcal{R}$ is an idempotent, $m=\left[\begin{array}{ll}a & b \\ 0 & 0\end{array}\right]_{p}$, then $m \in \mathcal{R}^{\#}$ if and only if $a \in \mathcal{R}^{\#}$ and $b=a a^{\#} b$. In this case,

$$
m^{\#}=\left[\begin{array}{cc}
a^{\#} & \left(a^{\#}\right)^{2} b \\
0 & 0
\end{array}\right]_{p} .
$$

Remark 2.4. The hypothesis " $c$ is Drazin invertible" in item (i) of Lemma 2.1 can be changed by " $a$ is Drazin invertible". The sketch of the proof is as follows: From (2.3), (2.4), and $m^{\#} m^{2}=m$ we get $u a^{2}=a$ and $w a^{2}=0$. Hence $r(a) \leq 1$, and the Drazin invertibility of $a$ leads to $i(a)=1$. Thus, $a \in \mathcal{R}^{\#}$ and so, $u a=a a^{\#}$ and $w a=0$. From $m^{\#}=\left(m^{\#}\right)^{2} m$ we get $w=w u a$; which in conjunction with $u a=a a^{\#}$ and $w a=0$ yields $w=0$. The proof finishes now as in Lemma 2.1.

REMARK 2.5. There is a version of Lemma 2.1 when the zero element is located at the "north-east" position. Assume that all the hypothesis of Lemma 2.1 hold except that $m=\left[\begin{array}{ll}a & 0 \\ b & c\end{array}\right]_{p}$. Since $\left[\begin{array}{ll}a & 0 \\ b & c\end{array}\right]_{p}=\left[\begin{array}{ll}c & b \\ 0 & a\end{array}\right]_{\bar{p}}$, we get

$$
\begin{aligned}
m^{\#} & =\left[\begin{array}{cc}
c^{\#} & \left(c^{\#}\right)^{2} b a^{\pi}+c^{\pi} b\left(a^{\#}\right)^{2}-c^{\#} b a^{\#} \\
0 & a^{\#}
\end{array}\right]_{\bar{p}} \\
& =\left[\begin{array}{cc}
a^{\#} & 0 \\
\left(c^{\#}\right)^{2} b a^{\pi}+c^{\pi} b\left(a^{\#}\right)^{2}-c^{\#} b a^{\#} & c^{\#}
\end{array}\right]_{p}
\end{aligned}
$$

and $c^{\pi} b a^{\pi}=0$.

Lemma 2.6. Let $\mathcal{R}$ be a unitary ring and $m \in \mathcal{R}$. If $p \in \mathcal{R}$ is an idempotent and $m=\left[\begin{array}{cc}a & 0 \\ 0 & c\end{array}\right]_{p}$, then $m \in \mathcal{R}^{\#}$ if and only if $a, c \in \mathcal{R}^{\#}$. In this case, one has $m^{\#}=\left[\begin{array}{cc}a^{\#} & 0 \\ 0 & c^{\#}\end{array}\right]_{p}$. 
Proof. Assume that $m$ is group invertible. Let us write $m^{\#}=\left[\begin{array}{cc}x & y \\ z & t\end{array}\right]_{p}$. From $m^{2} m^{\#}=m$ and $m^{\#} m^{2}=m$ we get $a^{2} x=a=x a^{2}$ and $c^{2} t=c=t c^{2}$. By Theorem 1.1, we obtain that $a$ and $c$ are group invertible. Conversely, if $a$ and $c$ are group invertible, it is enough to apply Lemma 2.1 to get this Lemma.

LEMma 2.7. Let $\mathcal{R}$ be a unitary ring and $a, b \in \mathcal{R}$ such that $a, b$ are group invertible. If $a$ is represented as in (2.2) and

$$
b=\left[\begin{array}{ll}
b_{1} & b_{2} \\
b_{3} & b_{4}
\end{array}\right]_{a a^{\#}},
$$

then the following conditions are equivalent:

(i) $a b$ is group invertible.

(ii) $a b_{1}$ is group invertible and $a b_{2}=a b_{1}\left(a b_{1}\right)^{\#} a b_{2}$.

Under this equivalence, one has

$$
(a b)^{\#}=\left[\begin{array}{cc}
\left(a b_{1}\right)^{\#} & \left(\left(a b_{1}\right)^{\#}\right)^{2} a b_{2} \\
0 & 0
\end{array}\right]_{a a^{\#}} .
$$

Proof. It follows from Remark 2.3.

Let $\mathcal{R}$ be a unitary ring and $a, b \in \mathcal{R}$. If $a$ is group invertible and $b$ is represented as in (2.11), then some equalities that will be used many times in the sequel are

$$
b_{1} a a^{\#}=a a^{\#} b_{1}=b_{1} \quad \text { and } \quad a a^{\#} b_{2}=b_{2},
$$

since $b_{1}=p b p$ and $b_{2}=p b \bar{p}$, where $p=a a^{\#}$. In particular, observe that $a$ is invertible in the subring $p \mathcal{R} p, a^{\#}$ is the inverse of $a$ in $p \mathcal{R} p$, and $p$ is the unity in this subring. But, we will avoid the use of the subring $p \mathcal{R} p$ along the paper by utilizing the equalities (2.13).

LEMma 2.8. Let $\mathcal{R}$ be a unitary ring and $a, b \in \mathcal{R}$ such that $a, b, a b$ are group invertible. If $b$ is represented as in (2.11), then $\left(a b_{1}\right)^{\#} a b_{2}=0$ if and only if $b_{2}=0$.

Proof. Let us assume that $\left(a b_{1}\right)^{\#} a b_{2}=0$ holds. Lemma 2.7 and the group invertibility of $a b$ yield $a b_{2}=a b_{1}\left(a b_{1}\right)^{\#} a b_{2}=0$, which implies $b_{2}=0$ in view of the second equality of (2.13). The converse is trivial.

LEMma 2.9. Let $\mathcal{R}$ be a unitary ring and $a, b \in \mathcal{R}$ such that $a, b, a b$ are group invertible. If a is represented as in (2.2),

$$
b=\left[\begin{array}{ll}
b_{1} & b_{2} \\
b_{3} & b_{4}
\end{array}\right]_{a a^{\#}}, \quad b^{\#}=\left[\begin{array}{ll}
c_{1} & c_{2} \\
c_{3} & c_{4}
\end{array}\right]_{a a^{\#}},
$$

and $b_{1}$ or $b_{4}$ are Drazin invertible, then the following conditions are equivalent: 
(i) $(a b)^{\#}=b^{\#} a^{\#}$.

(ii) $\left(a b_{1}\right)^{\#}=c_{1} a^{\#},\left(\left(a b_{1}\right)^{\#}\right)^{2} a b_{2}=0$, and $c_{3}=0$.

(iii) $b_{1}$ is group invertible, $b_{2}=b_{3}=0$, and $\left(a b_{1}\right)^{\#}=b_{1}^{\#} a^{\#}$.

Proof. Let us denote $p=a a^{\#}$.

(i) $\Leftrightarrow$ (ii): We apply Lemma 2.7. Combining (2.2), (2.12), and (2.14), it is easy to conclude that

$$
(a b)^{\#}=b^{\#} a^{\#} \quad \Leftrightarrow \quad\left(a b_{1}\right)^{\#}=c_{1} a^{\#}, \quad\left(\left(a b_{1}\right)^{\#}\right)^{2} a b_{2}=0, \quad c_{3} a^{\#}=0 .
$$

From (2.14) we have $c_{3}=\bar{p} b^{\#} p$. Thus, $c_{3} a^{\#}=\bar{p} b^{\#} p a^{\#}=\bar{p} b^{\#} a a^{\#} a^{\#}=\bar{p} b^{\#} a^{\#}$. If $c_{3} a^{\#}=0$, then $c_{3}=\bar{p} b^{\#} p=\bar{p} b^{\#} a^{\#} a=0$. Trivially, if $c_{3}=0$, then $c_{3} a^{\#}=0$. Therefore, the equivalence between items (i) and (ii) of the theorem has been proved.

(ii) $\Rightarrow$ (iii): By premultiplying $\left(\left(a b_{1}\right)^{\#}\right)^{2} a b_{2}=0$ by $a b_{1}$, we get $\left(a b_{1}\right)^{\#} a b_{2}=0$. Since $a b$ is group invertible, Lemma 2.8 yields $b_{2}=0$. Thus, $b=\left[\begin{array}{ll}b_{1} & 0 \\ b_{3} & b_{4}\end{array}\right]_{p}$. Since $b$ is group invertible and $b_{1}$ or $b_{4}$ are Drazin invertible, by using Lemma 2.1 and the representation of $b^{\#}$ given in (2.14), we get $c_{2}=0$. Recall that one hypothesis of (ii) is $c_{3}=0$. Therefore $b^{\#}=\left[\begin{array}{cc}c_{1} & 0 \\ 0 & c_{4}\end{array}\right]_{p}$. By Lemma 2.6 applied for $m=b^{\#}$, we get $b=\left(b^{\#}\right)^{\#}=\left[\begin{array}{cc}c_{1}^{\#} & 0 \\ 0 & c_{4}^{\#}\end{array}\right]_{p}$. So, $b_{3}=0$ and $b_{1}=c_{1}^{\#}$, and this proves (iii).

The implication (iii) $\Rightarrow$ (i) is trivial.

3. Reverse order law for the group inverse in rings. Now, we state the main result of this paper. Observe that if $a, b$ are elements in a ring such that $a$ is group invertible and $b$ is represented as in (2.11), then $b_{1}=a a^{\#} b a a^{\#}$ and $b_{4}=a^{\pi} b a^{\pi}$, which makes the connection between Lemma 2.9 and next result clearer.

TheOREM 3.1. Let $a, b$ be elements in a unitary ring $\mathcal{R}$ such that $a, b$ are group invertible. If $a a^{\#} b a a^{\#}$ or $a^{\pi} b a^{\pi}$ are Drazin invertible, then the following statements are equivalent:

(i) $a b \in \mathcal{R}^{\#}$ and $(a b)^{\#}=b^{\#} a^{\#}$.

(ii) $a b \in \mathcal{R}^{\#},(a b)^{\#}=b^{\#} a^{\#} a b b^{\#} a^{\#}, a a^{\#} b a^{\pi}=0$, and $a^{\pi} b a a^{\#}=0$.

(iii) $a b \in \mathcal{R}^{\#},(a b)^{\#}=b^{\#} a^{\#} a b b^{\#} a^{\#}$, and $a^{\pi} b a a^{\#}=0$.

(iv) $a^{\#} a b, a b b^{\#} \in \mathcal{R}^{\#},\left(a^{\#} a b\right)^{\#}=b^{\#} a^{\#} a$, and $\left(a b b^{\#}\right)^{\#}=b b^{\#} a^{\#}$.

(v) $a b, a^{\#} a b b^{\#} \in \mathcal{R}^{\#},(a b)^{\#}=b^{\#}\left(a^{\#} a b b^{\#}\right)^{\#} a^{\#},\left(a^{\#} a b b^{\#}\right)^{\#}=b b^{\#} a^{\#} a$.

(vi) $a b, a^{\#} a b \in \mathcal{R}^{\#},(a b)^{\#}=\left(a^{\#} a b\right)^{\#} a^{\#}$, and $\left(a^{\#} a b\right)^{\#}=b^{\#} a^{\#} a$.

(vii) $a b, a b b^{\#} \in \mathcal{R}^{\#},(a b)^{\#}=b^{\#}\left(a b b^{\#}\right)^{\#},\left(a b b^{\#}\right)^{\#}=b b^{\#} a^{\#}$.

(viii) $a b \in \mathcal{R}^{\#}, a b(a b)^{\#}=a b b^{\#} a^{\#}=b^{\#} a^{\#} a b, a^{\pi} b a a^{\#}=0$.

(ix) $a b \in \mathcal{R}^{\#},\left(b^{\#} a^{\#}\right) \mathcal{R} \subseteq(a b)^{\#} \mathcal{R},\left(b^{\#} a^{\#}\right)^{\circ} \subseteq\left((a b)^{\#}\right)^{\circ}$, and $a b b^{\#}=b b^{\#} a$. 
Proof. If we write $a$ as in (2.2) and we denote $p=a a^{\#}$, then we can represent $b$ and $b^{\#}$ as in (2.14).

(i) $\Rightarrow$ (ii): Observe that $(a b)^{\#}=(a b)^{\#} a b(a b)^{\#}=b^{\#} a^{\#} a b b^{\#} a^{\#}$. Lemma 2.9 yields $b_{2}=b_{3}=0$, which is equivalent to say $a a^{\#} b a^{\pi}=a^{\pi} b a a^{\#}=0$.

(ii) $\Rightarrow$ (iii) is evident.

(iii) $\Rightarrow$ (i): Notice that $b_{3}=a^{\pi} b a a^{\#}=0$. We can apply Lemma 2.1 to get $c_{3}=0$, $b_{1}^{\#}=c_{1}$, and $b_{4}^{\#}=c_{4}$. By employing $b=\left[\begin{array}{cc}b_{1} & b_{2} \\ 0 & b_{4}\end{array}\right]_{p}, b^{\#}=\left[\begin{array}{cc}b_{1}^{\#} & c_{2} \\ 0 & b_{4}^{\#}\end{array}\right]_{p},(2.13)$, and the representation of $a$ given in (2.2), after a straightforward computation we get

$$
b^{\#} a^{\#} a b b^{\#} a^{\#}=\left[\begin{array}{cc}
b_{1}^{\#} a^{\#} & 0 \\
0 & 0
\end{array}\right]_{p} .
$$

From $(a b)^{\#}=b^{\#} a^{\#} a b b^{\#} a^{\#}$, it follows that $\left(a b_{1}\right)^{\#}=b_{1}^{\#} a^{\#}$ and $\left(\left(a b_{1}\right)^{\#}\right)^{2} a b_{2}=0$. By the equivalence (i) $\Leftrightarrow$ (ii) of Lemma 2.9 we get $(a b)^{\#}=b^{\#} a^{\#}$.

(i) $\Rightarrow$ (iv): Since $(a b)^{\#}=b^{\#} a^{\#}$, from the equivalence (i) $\Rightarrow$ (iii) of Lemma 2.9, we deduce that $b=\left[\begin{array}{cc}b_{1} & 0 \\ 0 & b_{4}\end{array}\right]_{p}, a b_{1}, b_{1} \in \mathcal{R}^{\#}$, and $\left(a b_{1}\right)^{\#}=b_{1}^{\#} a^{\#}$. It is evident from (2.13) that

$$
a^{\#} a b=\left[\begin{array}{cc}
b_{1} & 0 \\
0 & 0
\end{array}\right]_{p} \quad \text { and } \quad b^{\#} a^{\#} a=\left[\begin{array}{cc}
b_{1}^{\#} & 0 \\
0 & 0
\end{array}\right]_{p} .
$$

Hence, $a^{\#} a b$ is group invertible and $\left(a^{\#} a b\right)^{\#}=b^{\#} a^{\#} a$. Now, let us prove that $a b_{1} b_{1}^{\#}$ is group invertible and $\left(a b_{1} b_{1}^{\#}\right)^{\#}=b_{1} b_{1}^{\#} a^{\#}$. To this end, we shall apply the uniqueness of the group inverse and $p b_{1}=b_{1} p=b_{1}, p b_{1}^{\#}=b_{1}^{\#} p=b_{1}^{\#}$. Let $x=a b_{1} b_{1}^{\#}$ and $y=b_{1} b_{1}^{\#} a^{\#}$. Now,

$$
x y=a b_{1} b_{1}^{\#} a^{\#}=a b_{1}\left(a b_{1}\right)^{\#}=\left(a b_{1}\right)^{\#} a b_{1}=b_{1}^{\#} a^{\#} a b_{1}=b_{1}^{\#} p b_{1}=b_{1}^{\#} b_{1}
$$

and

$$
y x=b_{1} b_{1}^{\#} p b_{1} b_{1}^{\#}=b_{1} b_{1}^{\#} b_{1} b_{1}^{\#}=b_{1}^{\#} b_{1}
$$

prove that $x y=y x$. Also we have

$$
x y x=x(y x)=a b_{1} b_{1}^{\#} b_{1} b_{1}^{\#}=a b_{1} b_{1}^{\#}=x
$$

and

$$
y x y=(y x) y=b_{1} b_{1}^{\#} b_{1} b_{1}^{\#} a^{\#}=b_{1} b_{1}^{\#} a^{\#}=y .
$$


Since

$$
a b b^{\#}=\left[\begin{array}{cc}
a b_{1} b_{1}^{\#} & 0 \\
0 & 0
\end{array}\right]_{p} \quad \text { and } \quad b b^{\#} a^{\#}=\left[\begin{array}{cc}
b_{1} b_{1}^{\#} a^{\#} & 0 \\
0 & 0
\end{array}\right]_{p}
$$

we get that $a b b^{\#}$ is group invertible and $\left(a b b^{\#}\right)^{\#}=b b^{\#} a^{\#}$.

(iv) $\Rightarrow(\mathrm{i})$ : We have

$$
a^{\#} a b=\left[\begin{array}{cc}
b_{1} & b_{2} \\
0 & 0
\end{array}\right]_{p}
$$

By Remark 2.3 and since $a^{\#} a b \in \mathcal{R}^{\#}$, we get that $b_{1} \in \mathcal{R}^{\#}, b_{2}=b_{1} b_{1}^{\#} b_{2}$, and

$$
\left(a^{\#} a b\right)^{\#}=\left[\begin{array}{cc}
b_{1}^{\#} & \left(b_{1}^{\#}\right)^{2} b_{2} \\
0 & 0
\end{array}\right]_{p} .
$$

By using $\left(a^{\#} a b\right)^{\#}=b^{\#} a^{\#} a$ one gets $b_{1}^{\#}=c_{1},\left(b_{1}^{\#}\right)^{2} b_{2}=0$, and $0=c_{3}$. Now we have $b_{2}=b_{1} b_{1}^{\#} b_{2}=b_{1}^{2}\left(b_{1}^{\#}\right)^{2} b_{2}=0$. Since $b^{\#}$ is group invertible, $c_{1}$ is Drazin invertible (because $c_{1}=b_{1}^{\#}$ ), and $c_{3}=0$, Lemma 2.1 allows us to get $b_{3}=0$. Thus, $b=\left[\begin{array}{cc}b_{1} & 0 \\ 0 & b_{4}\end{array}\right]_{p}$, and then

$$
\left(a b b^{\#}\right)^{\#}=\left[\begin{array}{cc}
\left(a b_{1} b_{1}^{\#}\right)^{\#} & 0 \\
0 & 0
\end{array}\right]_{p} \quad \text { and } \quad b b^{\#} a^{\#}=\left[\begin{array}{cc}
b_{1} b_{1}^{\#} a^{\#} & 0 \\
0 & 0
\end{array}\right]_{p} .
$$

Since $\left(a b b^{\#}\right)^{\#}=b b^{\#} a^{\#}$, we get $\left(a b_{1} b_{1}^{\#}\right)^{\#}=b_{1} b_{1}^{\#} a^{\#}$. In particular, $a b_{1} b_{1}^{\#}$ and $b_{1} b_{1}^{\#} a^{\#}$ commute. Hence $a b_{1} b_{1}^{\#} b_{1} b_{1}^{\#} a^{\#}=b_{1} b_{1}^{\#} a^{\#} a b_{1} b_{1}^{\#}$, which reduces in view of (2.13) to $a b_{1} b_{1}^{\#} a^{\#}=b_{1} b_{1}^{\#}$. Now we prove that $a b_{1}$ is group invertible and $\left(a b_{1}\right)^{\#}=b_{1}^{\#} a^{\#}$. Let $x=a b_{1}$ and $y=b_{1}^{\#} a^{\#}$. From $a b_{1} b_{1}^{\#} a^{\#}=b_{1} b_{1}^{\#}$ we get $x y=y x$. Now, $x y x=x(y x)=$ $a b_{1} b_{1} b_{1}^{\#}=a b_{1}=x$ and $y x y=(y x) y=b_{1} b_{1}^{\#} b_{1}^{\#} a^{\#}=b_{1}^{\#} a^{\#}=y$. The equivalence (i) $\Leftrightarrow$ (iii) of Lemma 2.9 allows us to prove that $a b$ is group invertible and $(a b)^{\#}=b^{\#} a^{\#}$.

$(\mathrm{i}) \Rightarrow(\mathrm{v})$ : From the hypothesis and Lemma 2.9 we get $b_{2}=b_{3}=0, a b_{1}, b_{1} \in \mathcal{R}^{\#}$, and $\left(a b_{1}\right)^{\#}=b_{1}^{\#} a^{\#}$. Let us recall that any idempotent is group invertible and its group inverse is itself, hence $\left(b_{1} b_{1}^{\#}\right)^{\#}=b_{1} b_{1}^{\#}$. From $(2.13)$ we have

$$
a^{\#} a b b^{\#}=\left[\begin{array}{cc}
a^{\#} a b_{1} b_{1}^{\#} & 0 \\
0 & 0
\end{array}\right]_{p}=\left[\begin{array}{cc}
b_{1} b_{1}^{\#} & 0 \\
0 & 0
\end{array}\right]_{p}
$$

hence $a^{\#} a b b^{\#} \in \mathcal{R}^{\#}$ and $\left(a^{\#} a b b^{\#}\right)^{\#}=b_{1} b_{1}^{\#} ;$ which yields

$$
\begin{aligned}
b^{\#}\left(a^{\#} a b b^{\#}\right)^{\#} a^{\#} & =\left[\begin{array}{cc}
b_{1}^{\#} b_{1} b_{1}^{\#} a^{\#} & 0 \\
0 & 0
\end{array}\right]_{p} \\
& =\left[\begin{array}{cc}
b_{1}^{\#} a^{\#} & 0 \\
0 & 0
\end{array}\right]_{p}=\left[\begin{array}{cc}
\left(a b_{1}\right)^{\#} & 0 \\
0 & 0
\end{array}\right]_{p}=(a b)^{\#} .
\end{aligned}
$$


Also we have $\left(a^{\#} a b b^{\#}\right)^{\#}=b b^{\#} a^{\#} a$.

(v) $\Rightarrow$ (i): Obviously, $(a b)^{\#}=b^{\#}\left(a^{\#} a b b^{\#}\right)^{\#} a^{\#}=b^{\#} b b^{\#} a^{\#} a a^{\#}=b^{\#} a^{\#}$.

(i) $\Rightarrow$ (vi): Since we have proved (i) $\Rightarrow$ (iv), we can use also (iv). Now, $(a b)^{\#}=$ $b^{\#} a^{\#}=b^{\#} a^{\#} a a^{\#}=\left(a^{\#} a b\right)^{\#} a^{\#}$.

$\left(\right.$ vi) $\Rightarrow$ (i): It is easy to see $(a b)^{\#}=\left(a^{\#} a b\right)^{\#} a^{\#}=b^{\#} a^{\#} a a^{\#}=b^{\#} a^{\#}$.

(i) $\Rightarrow$ (vii): We can use (iv), and therefore, $(a b)^{\#}=b^{\#} a^{\#}=b^{\#} b b^{\#} a^{\#}=$ $b^{\#}\left(a b b^{\#}\right)^{\#}$.

(vii) $\Rightarrow$ (i): Notice that $(a b)^{\#}=b^{\#}\left(a b b^{\#}\right)^{\#}=b^{\#} b b^{\#} a^{\#}=b^{\#} a^{\#}$.

(i) $\Rightarrow$ (viii): We will use the equivalence (i) $\Rightarrow$ (iii) of Lemma 2.9. The equality $a b(a b)^{\#}=a b b^{\#} a^{\#}$ is obvious from the hypothesis, and $a^{\pi} b a a^{\#}=0$ follows from $b_{3}=0$. Now, we have $a b(a b)^{\#}=(a b)^{\#} a b=b^{\#} a^{\#} a b$.

(viii) $\Rightarrow$ (i): Observe that from $a^{\pi} b a a^{\#}=0$, we get $b_{3}=0$. Thus, $b=\left[\begin{array}{cc}b_{1} & b_{2} \\ 0 & b_{4}\end{array}\right]_{p}$. From (2.12) and $a b(a b)^{\#}=a b b^{\#} a^{\#}$, we get $\left(a b_{1}\right)^{\#} a b_{2}=0$. Lemma 2.8 leads to $b_{2}=0$. Further, from $a b b^{\#} a^{\#}=b^{\#} a^{\#} a b$, we get $a b_{1} b_{1}^{\#} a^{\#}=b_{1}^{\#} b_{1}$. This implies that $b_{1}^{\#} a^{\#}$ obeys the equations to be the group inverse of $a b_{1}$. Hence $\left(a b_{1}\right)^{\#}=b_{1}^{\#} a^{\#}$. Then (i) holds.

(i) $\Rightarrow$ (ix): Since $(a b)^{\#}=b^{\#} a^{\#}$, we have $a b b^{\#} a^{\#}=b^{\#} a^{\#} a b$, which by using Lemma 2.9 reduces to $a b_{1} b_{1}^{\#} a^{\#}=b_{1} b_{1}^{\#}$. A postmultiplication by $a$ yields $a b_{1} b_{1}^{\#}=$ $b_{1} b_{1}^{\#} a$, which is equivalent to $a b b^{\#}=b b^{\#} a$.

(ix) $\Rightarrow$ (i): Observe that the expression of $(a b)^{\#}$ given in (2.12) can be used. Hence $a^{\pi} \in\left(b^{\#} a^{\#}\right)^{\circ} \subseteq\left[(a b)^{\#}\right]^{\circ}$ implies $(a b)^{\#} a^{\pi}=0$, which leads to $\left(\left(a b_{1}\right)^{\#}\right)^{2} a b_{2}=0$, or equivalently, $\left(a b_{1}\right)^{\#} a b_{2}=0$. Lemma 2.8 proves $b_{2}=0$. Hence (2.12) reduces to $(a b)^{\#}=\left[\begin{array}{cc}\left(a b_{1}\right)^{\#} & 0 \\ 0 & 0\end{array}\right]_{p}$. Lemma 2.1 implies that $b_{1}^{\#}=c_{1}$ and $b_{4}^{\#}=c_{4}$.

Since $b^{\#} a^{\#} \in\left(b^{\#} a^{\#}\right) \mathcal{R} \subseteq(a b)^{\#} \mathcal{R}$, there exists $u \in \mathcal{R}$ such that

$$
\left[\begin{array}{ll}
c_{1} & c_{2} \\
c_{3} & c_{4}
\end{array}\right]_{p}\left[\begin{array}{cc}
a^{\#} & 0 \\
0 & 0
\end{array}\right]_{p}=\left[\begin{array}{cc}
\left(a b_{1}\right)^{\#} & 0 \\
0 & 0
\end{array}\right]_{p}\left[\begin{array}{ll}
\text { pup } & p u \bar{p} \\
\bar{p} u p & \bar{p} u \bar{p}
\end{array}\right]_{p} .
$$

Therefore, $0=c_{3} a^{\#}$, which in conjunction of $c_{3}=\bar{p} b^{\#} p$, implies $0=c_{3} a^{\#} a=c_{3} p=$ $c_{3}$. Lemma 2.1 yields $b_{3}=0$.

By Lemma 2.9 , to prove $(a b)^{\#}=b^{\#} a^{\#}$, it is enough to prove $\left(a b_{1}\right)^{\#}=b_{1}^{\#} a^{\#}$. From $b=\left[\begin{array}{cc}b_{1} & 0 \\ 0 & b_{4}\end{array}\right]_{p}$ and $a b b^{\#}=b b^{\#} a$, we get $a b_{1} b_{1}^{\#}=b_{1} b_{1}^{\#} a$, which by (2.13), leads to $a b_{1} b_{1}^{\#} a^{\#}=b_{1} b_{1}^{\#} a a^{\#}=b_{1} b_{1}^{\#}=b_{1} a a^{\#} b_{1}^{\#}$. The equalities

$$
\left(a b_{1}\right)\left(b_{1}^{\#} a^{\#}\right)\left(a b_{1}\right)=a b_{1} \quad \text { and } \quad\left(b_{1}^{\#} a^{\#}\right)\left(a b_{1}\right)\left(b_{1}^{\#} a^{\#}\right)=b_{1}^{\#} a^{\#}
$$


are trivial to prove. Hence, by the uniqueness of the group inverse, $\left(a b_{1}\right)^{\#}=b_{1}^{\#} a^{\#}$.

REMARK 3.2. Let $\mathcal{R}$ be a unitary ring and $a, b \in \mathcal{R}^{\#}$. The following two equivalences were proved in [13, Theorems 2.4 and 2.5]:

- $a^{\#} a b \in \mathcal{R}^{\#}$ and $\left(a^{\#} a b\right)^{\#}=b^{\#} a a^{\#} \Leftrightarrow a^{\#} a b=b a^{\#} a$.

- $a b^{\#} b \in \mathcal{R}^{\#}$ and $\left(a b b^{\#}\right)^{\#}=b b^{\#} a^{\#} \Leftrightarrow a b b^{\#}=b^{\#} b a$.

The following result was established in [4] when the setting is the Banach algebra of all bounded linear operators on a Hilbert space. However, we will establish this result in rings by using only algebraic techniques. Notice that, in particular, involution and norms will not be used (which were employed in [4]).

TheOREM 3.3. Let $\mathcal{R}$ be a unitary ring and $a, b \in \mathcal{R}^{\#}$ such that $a b \in \mathcal{R}^{\#}$.

(i) If $a a^{\#} b \in \mathcal{R}^{\#}$, then $(a b)^{\#}=\left(a a^{\#} b\right)^{\#} a^{\#} \Leftrightarrow a b a^{\pi}=0$ and $\left[a,\left(a a^{\#} b\right)^{\pi}\right]=0$.

(ii) If $a b b^{\#} \in \mathcal{R}^{\#}$, then $(a b)^{\#}=b^{\#}\left(a b b^{\#}\right)^{\#} \Leftrightarrow b^{\pi} a b=0$ and $\left[b,\left(a b b^{\#}\right)^{\pi}\right]=0$.

Proof. Assume that $(a b)^{\#}=\left(a a^{\#} b\right)^{\#} a^{\#}$. From (2.12) and (3.2) we get $\left(a b_{1}\right)^{\#}=$ $b_{1}^{\#} a^{\#}$ and $\left(a b_{1}\right)^{\#} a b_{2}=0$. By using Lemma 2.8 we get $0=b_{2}=p b \bar{p}$, which yields $0=a b a^{\pi}$. Also, since any group invertible element commutes with its group inverse, $\left(a b_{1}\right)^{\#}=b_{1}^{\#} a^{\#}$ implies $a b_{1} b_{1}^{\#} a^{\#}=b_{1}^{\#} a^{\#} a b_{1}$, which reduces in view of (2.13) to $a b_{1} b_{1}^{\#} a^{\#}=b_{1}^{\#} b_{1}$, which by a postmultiplication by $a$, simplifies to $a b_{1} b_{1}^{\#}=b_{1}^{\#} b_{1} a$. By using this last equality, (3.1), (3.2), and $b_{2}=0$ we get that $\left(a a^{\#} b\right)\left(a a^{\#} b\right)^{\#}$ commutes with $a$, which is equivalent to $\left[a,\left(a a^{\#} b\right)^{\pi}\right]=0$.

Let us prove the converse. Observe that $b_{2}=a^{\#} a b a^{\pi}=0$. By using the representations (3.1), (3.2), and the hypotheses we get $a b_{1} b_{1}^{\#}=b_{1} b_{1}^{\#} a$. By the definition of the group inverse we easily get $\left(a b_{1}\right)^{\#}=b_{1}^{\#} a^{\#}$. Now, it is trivial to get $(a b)^{\#}=\left(a a^{\#} b\right)^{\#} a^{\#}$.

(ii) Let $q=b b^{\#}$. The proof is easy if we proceed as in previous item and use the following representations:

$$
\begin{gathered}
a=\left[\begin{array}{ll}
a_{1} & a_{2} \\
a_{3} & a_{4}
\end{array}\right]_{q}, \quad b=\left[\begin{array}{ll}
b & 0 \\
0 & 0
\end{array}\right]_{q}, \quad b^{\#}=\left[\begin{array}{cc}
b^{\#} & 0 \\
0 & 0
\end{array}\right]_{q}, \quad a b=\left[\begin{array}{ll}
a_{1} b & 0 \\
a_{3} b & 0
\end{array}\right]_{q}, \\
(a b)^{\#}=\left[\begin{array}{cc}
\left(a_{1} b\right)^{\#} & 0 \\
a_{3} b\left(\left(a_{1} b\right)^{\#}\right)^{2} & 0
\end{array}\right]_{q}, \quad a b b^{\#}=\left[\begin{array}{ll}
a_{1} & 0 \\
a_{3} & 0
\end{array}\right]_{q}, \quad\left(a b b^{\#}\right)^{\#}=\left[\begin{array}{cc}
a_{1}^{\#} & 0 \\
a_{3}\left(a_{1}^{\#}\right)^{2} & 0
\end{array}\right]_{q} .
\end{gathered}
$$

We omit the details.

This last result appeared in [13] (Theorem 2.2 (i),(iii) and Theorem 2.3 (i), (iii)) with another proof.

COROLlary 3.4. Let $\mathcal{R}$ be a unitary ring and $a, b \in \mathcal{R}$ be such that $a, b \in \mathcal{R}^{\#}$ 
and $a a^{\#} b a a^{\#}$ or $a^{\pi} b a^{\pi}$ are Drazin invertible. Then the following statements are equivalent:

(i) $a b \in \mathcal{R}^{\#}$ and $(a b)^{\#}=b^{\#} a^{\#}$.

(ii) $\left[a^{\pi}, b\right]=0,\left[a, b^{\pi}\right]=0$.

(iii) $a b, a a^{\#} b \in \mathcal{R}^{\#}, a b a^{\pi}=0,\left[a,\left(a^{\#} a b\right)^{\pi}\right]=0,\left[a^{\pi}, b\right]=0$.

(iv) $a b, a b b^{\#} \in \mathcal{R}^{\#}, b^{\pi} a b=0,\left[b,\left(a b b^{\#}\right)^{\pi}\right]=0,\left[a, b^{\pi}\right]=0$.

Proof. The equivalence of item (iv) Theorem 3.1 and (ii) of this corollary has been noticed in Remark 3.2. The equivalence of item (vi) Theorem 3.1 and (iii) of this corollary has been proved in Theorem 3.3. In the same way, we have that item (vii) of Theorem 3.1 and (iv) of this corollary are equivalent.

4. Reverse order law for the group inverse in rings with involution. In this section we will assume that the unitary ring $\mathcal{R}$ has an involution. Let us recall that if the ring $\mathcal{R}$ has an involution, then the Moore-Penrose invertibility in $\mathcal{R}$ can be defined.

Taking involution in the definition of the group inverse we immediately have $a \in \mathcal{R}^{\#} \Leftrightarrow a^{*} \in \mathcal{R}^{\#}$ and in this case, $\left(a^{*}\right)^{\#}=\left(a^{\#}\right)^{*}$.

As we remarked, if an element a of a ring $\mathcal{R}$ with involution is EP, then $a \in \mathcal{R}^{\#}$ and $a^{\dagger}=a^{\#}$. There is a version of (2.1) for this class of elements (see [3, Theorem $2.1])$ :

$a$ is $\mathrm{EP} \Leftrightarrow$ there is a projection $p \in \mathcal{R}$ such that $a+p \in \mathcal{R}^{-1}$ and $a p=p a=0$.

The representation (2.2) is also valid. But, observe that this representation preserves the involution since $a a^{\dagger}$ is self-adjoint. When (4.1) is applied to an EP matrix $A \in$ $\mathbb{C}^{n \times n}$, by writing the idempotent $A^{\pi}$ as $U\left(0 \oplus I_{k}\right) U^{*}$, where $U \in \mathbb{C}^{n \times n}$ is unitary (see e.g. [21, Theorem 5.4]), there exists a nonsingular matrix $B \in \mathbb{C}^{(n-k) \times(n-k)}$ such that $A=U(B \oplus 0) U^{*}$. Obviously, $A^{\dagger}=U\left(B^{-1} \oplus 0\right) U^{*}$.

For a complex matrix $A \in \mathbb{C}^{n \times m}$, the matrix $A^{*} A$ has been extensively studied (it appears, for example, when one uses a least squares technique). This matrix, evidently, is Hermitian (self-adjoint in the nomenclature used in this paper), hence it is unitarily diagonalizable, i.e. there exist a unitary matrix $U \in \mathbb{C}^{m \times m}$ and a diagonal matrix $D \in \mathbb{C}^{m \times m}$ such that $A^{*} A=U D U^{*}$. Hence $A^{*} A$ is group invertible. In next result, under the additional hypothesis " $A$ is EP" we see that the reverse order law for the group inverse holds for $A^{*} A$ in rings.

THEOREM 4.1. Let $\mathcal{R}$ be a unitary ring with involution. If $a \in \mathcal{R}$ is $E P$, then $a^{*} a$ is EP and $\left(a^{*} a\right)^{\#}=a^{\#}\left(a^{*}\right)^{\#}$.

Proof. Observe that $a a^{\#}=a^{\#} a$ is self-adjoint because $a$ is EP. We shall see that 
$a^{\#}\left(a^{*}\right)^{\#}$ satisfies the equations to be the group inverse of $a^{*} a$. Since

$$
\begin{gathered}
a^{*} a a^{\#}\left(a^{*}\right)^{\#}=a^{*}\left(a a^{\#}\right)^{*}\left(a^{\#}\right)^{*}=\left(a^{\#} a a^{\#} a\right)^{*}=\left(a^{\#} a\right)^{*}=a^{\#} a, \\
a^{\#}\left(a^{*}\right)^{\#} a^{*} a=a^{\#}\left(a^{\#}\right)^{*} a^{*} a=a^{\#}\left(a a^{\#}\right)^{*} a=a^{\#} a a^{\#} a=a^{\#} a,
\end{gathered}
$$

we get $\left[a^{*} a\right]\left[a^{\#}\left(a^{*}\right)^{\#}\right]=\left[a^{\#}\left(a^{*}\right)^{\#}\right]\left[a^{*} a\right]$. Now, we have

$$
a^{*} a a^{\#}\left(a^{*}\right)^{\#} a^{*} a=a^{\#} a a^{*} a=\left(a^{\#} a\right)^{*} a^{*} a=\left(a a^{\#} a\right)^{*} a=a^{*} a .
$$

The equality $a^{\#}\left(a^{*}\right)^{\#} a^{*} a a^{\#}\left(a^{*}\right)^{\#}=a^{\#}\left(a^{*}\right)^{\#}$ is easy to prove. Since $a^{*} a$ is self-adjoint and group invertible, then $a^{*} a$ is EP.

LEMma 4.2. Let $\mathcal{R}$ be a unitary ring with involution. If $a \in \mathcal{R}$ is $E P$, then $\left(a a^{*}\right)^{k}\left[\left(a a^{*}\right)^{\#}\right]^{k}=a a^{\#}$ for any $k \in \mathbb{N}$.

Proof. We shall use Theorem 4.1 and $\left(a a^{\#}\right)^{*}=a a^{\#}$ because $a$ is EP. The lemma holds for $k=1$ since

$$
\left(a a^{*}\right)\left(a a^{*}\right)^{\#}=a a^{*}\left(a^{\#}\right)^{*} a^{\#}=a\left(a^{\#} a\right)^{*} a^{\#}=a a^{\#} a a^{\#}=a a^{\#} .
$$

Now pick any $n \in \mathbb{N}$. Now, by the previous computation and the definition of the group inverse of $a a^{*}$ we have $\left(a a^{*}\right)^{n+1}\left[\left(a a^{*}\right)^{\#}\right]^{n+1}=a a^{*}\left(a a^{*}\right)^{\#}=a a^{\#}$.

THEOREM 4.3. Let $\mathcal{R}$ be a unitary ring with involution. If $a, b$ are EP, then the following statements are equivalent:

(i) $a b \in \mathcal{R}^{\#}$ and $(a b)^{\#}=b^{\#} a^{\#}$.

(ii) $\left(a^{\#}\right)^{*} b \in \mathcal{R}^{\#}$ and $\left(\left(a^{\#}\right)^{*} b\right)^{\#}=b^{\#} a^{*}$.

(iii) $a\left(b^{\#}\right)^{*} \in \mathcal{R}^{\#}$ and $\left(a\left(b^{\#}\right)^{*}\right)^{\#}=b^{*} a^{\#}$.

(iv) $a b, a^{*} a b \in \mathcal{R}^{\#}$ and $(a b)^{\#}=\left(a^{*} a b\right)^{\#} a^{*},\left(a^{*} a b\right)^{\#}=b^{\#}\left(a^{*} a\right)^{\#}$.

(v) $a b, a b b^{*} \in \mathcal{R}^{\#}$ and $(a b)^{\#}=b^{*}\left(a b b^{*}\right)^{\#},\left(a b b^{*}\right)^{\#}=\left(b b^{*}\right)^{\#} a^{\#}$.

(vi) $a b \in \mathcal{R}^{\#}$ and for any $m, n \in \mathbb{N} \cup\{0\},\left(a^{*} a\right)^{m+1}\left(b b^{*}\right)^{n+1} \in \mathcal{R}^{\#}$,

$$
\left[\left(a^{*} a\right)^{m+1}\left(b b^{*}\right)^{n+1}\right]^{\#}=\left[\left(b b^{*}\right)^{\#}\right]^{n+1}\left[\left(a^{*} a\right)^{\#}\right]^{m+1},
$$

and

$$
b^{*}\left(b b^{*}\right)^{n}\left[\left(a^{*} a\right)^{m+1}\left(b b^{*}\right)^{n+1}\right]^{\#}\left(a^{*} a\right)^{m} a^{*}=(a b)^{\#} .
$$

(vii) $a b \in \mathcal{R}^{\#}$ and exist $m, n \in \mathbb{N} \cup\{0\}$ such that $\left(a^{*} a\right)^{m+1}\left(b b^{*}\right)^{n+1} \in \mathcal{R}^{\#}$ and (4.2), (4.3) are satisfied.

Furthermore, under any of the previous conditions, one has that ab is EP.

Proof. Let us represent $a, a^{\#}$ as in (2.2) and $b, b^{\#}$ as in (2.14). Let $p=a a^{\#}$. Notice that since $a$ is EP, the idempotent $p$ is a projection and the representations (2.2) and (2.14) preserve the involution. 

that

(i) $\Rightarrow$ (ii): By Lemma 2.9 we have $b_{2}=b_{3}=0$ and $\left(a b_{1}\right)^{\#}=b_{1}^{\#} a^{\#}$. It is obvious

$$
\left(a^{\#}\right)^{*} b=\left[\begin{array}{cc}
\left(a^{\#}\right)^{*} b_{1} & 0 \\
0 & 0
\end{array}\right]_{p} \quad \text { and } \quad b^{\#} a^{*}=\left[\begin{array}{cc}
b_{1}^{\#} a^{*} & 0 \\
0 & 0
\end{array}\right]_{p} .
$$

We will prove that $b_{1}^{\#} a^{*}$ verifies the three conditions to be the group inverse of $\left(a^{\#}\right)^{*} b_{1}$. Since $\left(a b_{1}\right)^{\#}=b_{1}^{\#} a^{\#}$, in particular from (2.13) we have

$$
a b_{1} b_{1}^{\#} a^{\#}=b_{1}^{\#} a^{\#} a b_{1}=b_{1}^{\#} b_{1} .
$$

Observe that $b$ is EP and $b=\left[\begin{array}{cc}b_{1} & 0 \\ 0 & b_{4}\end{array}\right]_{p}$ imply that $b_{1}$ is also EP. Also, let us remark that since $a$ and $b_{1}$ are EP, then $a a^{\#}$ and $b_{1} b_{1}^{\#}$ are self-adjoint. From (4.5) we have

$$
\left[\left(a^{\#}\right)^{*} b_{1}\right]\left[b_{1}^{\#} a^{*}\right]=\left(a^{\#}\right)^{*}\left(b_{1} b_{1}^{\#}\right)^{*} a^{*}=\left[a b_{1} b_{1}^{\#} a^{\#}\right]^{*}=b_{1} b_{1}^{\#}
$$

and

$$
\left[b_{1}^{\#} a^{*}\right]\left[\left(a^{\#}\right)^{*} b_{1}\right]=b_{1}^{\#}\left(a^{*}\left(a^{\#}\right)^{*}\right) b_{1}=b_{1}^{\#}\left(a^{\#} a\right)^{*} b_{1}=b_{1}^{\#} a^{\#} a b_{1}=b_{1}^{\#} b_{1} .
$$

Now it is easy to prove

$$
\left[\left(a^{\#}\right)^{*} b_{1}\right]\left[b_{1}^{\#} a^{*}\right]\left[\left(a^{\#}\right)^{*} b_{1}\right]=\left(a^{\#}\right)^{*} b_{1} \quad \text { and } \quad\left[b_{1}^{\#} a^{*}\right]\left[\left(a^{\#}\right)^{*} b_{1}\right]\left[b_{1}^{\#} a^{*}\right]=b_{1}^{\#} a^{*},
$$

which yields that $\left(\left(a^{\#}\right)^{*} b_{1}\right)^{\#}=b_{1}^{\#} a^{*}$. Hence (4.4) proves that $\left(a^{\#}\right)^{*} b$ is group invertible and $\left(\left(a^{\#}\right)^{*} b\right)^{\#}=b^{\#} a^{*}$.

(ii) $\Rightarrow$ (i): Let $c=\left(a^{\#}\right)^{*}=\left(a^{*}\right)^{\#}$. As we have proved (i) $\Rightarrow$ (ii), we can use this implication. The hypotheses are $c b \in \mathcal{R}^{\#}$ and $(c b)^{\#}=b^{\#} c^{\#}$. Thus, $\left(c^{\#}\right)^{*} b \in \mathcal{R}^{\#}$ and $\left(\left(c^{\#}\right)^{*} b\right)^{\#}=b^{\#} c^{*}$. Hence, (i) holds.

(i) $\Leftrightarrow$ (iii): Let $x=b^{*}$ and $y=a^{*}$. By the previously proved equivalence (i) $\Leftrightarrow$ (ii) we have

$$
\begin{aligned}
& (a b)^{\#}=b^{\#} a^{\#} \Leftrightarrow\left[(a b)^{\#}\right]^{*}=\left[b^{\#} a^{\#}\right]^{*} \Leftrightarrow(x y)^{\#}=y^{\#} x^{\#} \Leftrightarrow\left[\left(x^{\#}\right)^{*} y\right]^{\#}=y^{\#} x^{*} \\
& \Leftrightarrow\left(y^{*} x^{\#}\right)^{\#}=x\left(y^{\#}\right)^{*} \Leftrightarrow\left(a\left(b^{\#}\right)^{*}\right)=b^{*} a^{\#} \text {. }
\end{aligned}
$$

(i) $\Rightarrow$ (iv): By Lemma 2.9, we get $b=\left[\begin{array}{cc}b_{1} & 0 \\ 0 & b_{4}\end{array}\right]_{p}$ and $b_{1}^{\#} a^{\#}=\left(a b_{1}\right)^{\#}$. By using the forms of $a$ and $b$, we get

$$
a^{*} a b=\left[\begin{array}{cc}
a^{*} a b_{1} & 0 \\
0 & 0
\end{array}\right]_{p} \quad \text { and } \quad b^{\#}\left(a^{*} a\right)^{\#}=\left[\begin{array}{cc}
b_{1}^{\#}\left(a^{*} a\right)^{\#} & 0 \\
0 & 0
\end{array}\right]_{p} .
$$

From the equivalence (i) $\Leftrightarrow$ (ix) of Theorem 3.1 we get $a b b^{\#}=b b^{\#} a$, or equivalently, $a b_{1} b_{1}^{\#}=b_{1}^{\#} b_{1} a$. We shall prove that $a^{*} a b_{1}$ is group invertible and $\left(a^{*} a b_{1}\right)^{\#}=$ 
$b_{1}^{\#}\left(a^{*} a\right)^{\#}$. To this end, we use Theorem 4.1 and $\left(a a^{\#}\right)^{*}=a a^{\#},\left(b_{1} b_{1}^{\#}\right)^{*}=b_{1} b_{1}^{\#}$. We have from $(2.13)$

$$
\begin{aligned}
a^{*} a b_{1} b_{1}^{\#}\left(a^{*} a\right)^{\#} & =a^{*}\left(a b_{1} b_{1}^{\#}\right) a^{\#}\left(a^{*}\right)^{\#}=a^{*} b_{1}^{\#} b_{1} a a^{\#}\left(a^{*}\right)^{\#}=a^{*}\left(b_{1}^{\#} b_{1}\right)^{*}\left(a^{\#}\right)^{*} \\
& =\left(a^{\#} b_{1}^{\#} b_{1} a\right)^{*}=\left(a^{\#} a b_{1}^{\#} b_{1}\right)^{*}=\left(b_{1}^{\#} b_{1}\right)^{*}=b_{1}^{\#} b_{1}
\end{aligned}
$$

and

$$
\begin{aligned}
b_{1}^{\#}\left(a^{*} a\right)^{\#} a^{*} a b_{1} & =b_{1}^{\#} a^{\#}\left(a^{*}\right)^{\#} a^{*} a b_{1} \\
& =b_{1}^{\#} a^{\#}\left(a a^{\#}\right)^{*} a b_{1}=b_{1}^{\#} a^{\#} a a^{\#} a b_{1}=b_{1}^{\#} a^{\#} a b_{1}=b_{1}^{\#} b_{1},
\end{aligned}
$$

which prove $\left[a^{*} a b_{1}\right]\left[b_{1}^{\#}\left(a^{*} a\right)^{\#}\right]=\left[b_{1}^{\#}\left(a^{*} a\right)^{\#}\right]\left[a^{*} a b_{1}\right]$. Now by using (4.6) we can easily check the two remaining equalities for the group inverse of $a^{*} a b_{1}$.

Furthermore, we have

$$
\begin{aligned}
\left(a^{*} a b_{1}\right)^{\#} a^{*} & =b_{1}^{\#}\left(a^{*} a\right)^{\#} a^{*} \\
& =b_{1}^{\#} a^{\#}\left(a^{\#}\right)^{*} a^{*}=b_{1}^{\#} a^{\#}\left(a a^{\#}\right)^{*}=b_{1}^{\#} a^{\#} a a^{\#}=b_{1}^{\#} a^{\#}=\left(a b_{1}\right)^{\#},
\end{aligned}
$$

which proves $\left(a^{*} a b\right)^{\#} a^{*}=(a b)^{\#}$.

(iv) $\Rightarrow$ (i) follows from

$$
\begin{aligned}
(a b)^{\#} & =\left(a^{*} a b\right)^{\#} a^{*} \\
& =b^{\#}\left(a^{*} a\right)^{\#} a^{*}=b^{\#} a^{\#}\left(a^{\#}\right)^{*} a^{*}=b^{\#} a^{\#}\left(a a^{\#}\right)^{*}=b^{\#} a^{\#} a a^{\#}=b^{\#} a^{\#} .
\end{aligned}
$$

(i) $\Leftrightarrow$ (v): It is enough to apply (i) $\Leftrightarrow$ (iv) by substituting $a \leftrightarrow b^{*}$ and $b \leftrightarrow a^{*}$.

(i) $\Rightarrow$ (vi): By Lemma 2.9 we have $b_{2}=b_{3}=0$. As we have established in the proof of (i) $\Rightarrow$ (iv), we can use

$$
a b_{1} b_{1}^{\#}=b_{1}^{\#} b_{1} a
$$

Taking adjoint of (4.7) and using that $b$ is EP we get

$$
b_{1} b_{1}^{\#} a^{*}=a^{*} b_{1}^{\#} b_{1} .
$$

We have for any $m, n \in \mathbb{N} \cup\{0\}$

$$
\left(a^{*} a\right)^{m+1}\left(b b^{*}\right)^{n+1}=\left[\begin{array}{cc}
\left(a^{*} a\right)^{m+1}\left(b_{1} b_{1}^{*}\right)^{n+1} & 0 \\
0 & 0
\end{array}\right]_{p}
$$

and

$$
\left[\left(b b^{*}\right)^{\#}\right]^{n+1}\left[\left(a^{*} a\right)^{\#}\right]^{m+1}=\left[\begin{array}{cc}
{\left[\left(b_{1} b_{1}^{*}\right)^{\#}\right]^{n+1}\left[\left(a^{*} a\right)^{\#}\right]^{m+1}} & 0 \\
0 & 0
\end{array}\right]_{p} .
$$


Observe that $\left(b_{1} b_{1}^{*}\right)^{n+1}\left[\left(b_{1} b_{1}^{*}\right)^{\#}\right]^{n+1}=b_{1} b_{1}^{*}\left(b_{1} b_{1}^{*}\right)^{\#}$ and by using Lemma 4.2 (for $b_{1}$ and $k=1$ ), we have $b_{1} b_{1}^{*}\left(b_{1} b_{1}^{*}\right)^{\#}=b_{1} b_{1}^{\#}$. From Lemma 4.2 (applied to $\left.a\right),(2.13)$, (4.7), and (4.8) we have

$$
\begin{aligned}
\left(a^{*} a\right)^{m+1}\left(b_{1} b_{1}^{*}\right)^{n+1}\left[\left(b_{1} b_{1}^{*}\right)^{\#}\right]^{n+1}\left[\left(a^{*} a\right)^{\#}\right]^{m+1} & =\left(a^{*} a\right)^{m+1} b_{1} b_{1}^{\#}\left[\left(a^{*} a\right)^{\#}\right]^{m+1} \\
& =b_{1} b_{1}^{\#}\left(a^{*} a\right)^{m+1}\left[\left(a^{*} a\right)^{\#}\right]^{m+1} \\
& =b_{1} b_{1}^{\#} a a^{\#} \\
& =b_{1} b_{1}^{\#} .
\end{aligned}
$$

By using (2.13) and Lemma 4.2 we trivially get

$$
\left[\left(b_{1} b_{1}^{*}\right)^{\#}\right]^{n+1}\left[\left(a^{*} a\right)^{\#}\right]^{m+1}\left(a^{*} a\right)^{m+1}\left(b_{1} b_{1}^{*}\right)^{n+1}=b_{1} b_{1}^{\#} .
$$

If we define $x=\left(a^{*} a\right)^{m+1}\left(b_{1} b_{1}^{*}\right)^{n+1}$ and $y=\left[\left(b_{1} b_{1}^{*}\right)^{\#}\right]^{n+1}\left[\left(a^{*} a\right)^{\#}\right]^{m+1}$, then equalities (4.9) and (4.10) prove $x y=y x$.

Now, the next computation

$$
b_{1} b_{1}^{\#}\left(b_{1} b_{1}^{*}\right)^{\#}=\left(b_{1} b_{1}^{\#}\right)^{*}\left(b_{1}^{\#}\right)^{*} b_{1}^{\#}=\left(b_{1}^{\#} b_{1} b_{1}^{\#}\right)^{*} b_{1}^{\#}=\left(b_{1}^{\#}\right)^{*} b_{1}^{\#}=\left(b_{1} b_{1}^{*}\right)^{\#}
$$

proves

$$
b_{1} b_{1}^{\#}\left[\left(b_{1} b_{1}^{*}\right)^{\#}\right]^{n+1}=\left[\left(b_{1} b_{1}^{*}\right)^{\#}\right]^{n+1} .
$$

Equalities (4.10) and (4.11) allow to get $y x y=y$.

From $b_{1} b_{1}^{*} b_{1} b_{1}^{\#}=b_{1} b_{1}^{*}\left(b_{1} b_{1}^{\#}\right)^{*}=b_{1}\left(b_{1} b_{1}^{\#} b_{1}\right)^{*}=b_{1} b_{1}^{*}$, we get

$$
\left(b_{1} b_{1}^{*}\right)^{q+1} b_{1} b_{1}^{\#}=\left(b_{1} b_{1}^{*}\right)^{n+1} .
$$

Equalities (4.10) and (4.12) prove $x y x=x$. Thus, $x, y \in \mathcal{R}^{\#}$ and $x^{\#}=y$. Hence we have proved

$$
\left[\left(a^{*} a\right)^{m+1}\left(b b^{*}\right)^{n+1}\right]^{\#}=\left[\left(b b^{*}\right)^{\#}\right]^{n+1}\left[\left(a^{*} a\right)^{\#}\right]^{m+1} .
$$

Finally, by Theorem 4.1

$$
\begin{aligned}
b^{*}\left(b b^{*}\right)^{n}\left[\left(a^{*} a\right)^{m+1}\left(b b^{*}\right)^{n+1}\right]^{\#}\left(a^{*} a\right)^{m} a^{*} & =b^{*}\left(b b^{*}\right)^{n}\left[\left(b b^{*}\right)^{\#}\right]^{n+1}\left[\left(a^{*} a\right)^{\#}\right]^{m+1}\left(a^{*} a\right)^{m} a^{*} \\
& =b^{*}\left(b b^{*}\right)^{\#}\left(a^{*} a\right)^{\#} a^{*} \\
& =b^{*}\left(b^{\#}\right)^{*} b^{\#} a^{\#}\left(a^{\#}\right)^{*} a^{*} \\
& =\left(b^{\#} b\right)^{*} b^{\#} a^{\#}\left(a a^{\#}\right)^{*}=b^{\#} b b^{\#} a^{\#} a a^{\#} \\
& =b^{\#} a^{\#}=(a b)^{\#} .
\end{aligned}
$$

(vi) $\Rightarrow$ (vii) is evident. 
(vii) $\Rightarrow$ (i): By the hypotheses we have $(a b)^{\#}=b^{*}\left(b b^{*}\right)^{\#}\left(a^{*} a\right)^{\#} a^{*}$. Furthermore, by Theorem 4.1 and the EP-ness of $b$ we have $b^{*}\left(b b^{*}\right)^{\#}=b^{*}\left(b^{\#}\right)^{*} b^{\#}=\left(b^{\#} b\right)^{*} b^{\#}=$ $b^{\#} b b^{\#}=b^{\#}$. In the same way we can prove $\left(a^{*} a\right)^{\#} a^{*}=a^{\#}$. Hence $(a b)^{\#}=b^{\#} a^{\#}$.

To prove that $a b$ is EP, observe that by the used representations of $a$ and $b$ we have $a b=\left[\begin{array}{cc}a b_{1} & 0 \\ 0 & 0\end{array}\right]_{p}$. Hence $a b$ is EP if and only if $a b_{1}$ is EP. From the proof of (i) $\Rightarrow$ (iii) we get $\left(a b_{1}\right)^{\#} a b_{1}=b_{1}^{\#} a^{\#} a b_{1}=b_{1}^{\#} b_{1}$ is self-adjoint. Hence $\left(a b_{1}\right)^{\#}=\left(a b_{1}\right)^{\dagger}$, and thus, $a b_{1}$ is EP.

Acknowledgments. The first and the second authors have been supported by the National Natural Science Foundation of China (11061005) and the Ministry of Education Science and Technology Key Project (210164). The third author has been supported by the Universidad Politécnica de Valencia (PAID-06-12).

We would like to thank two anonymous referees for their very detailed comments and valuable suggestions which greatly improved our presentation.

\section{REFERENCES}

[1] E. Arghiriade, Sur les matrices qui sont permutables avec leur inverse généralisée, Atti Accad. Naz. Lincei Rend. Cl. Sci. Fis. Mat. Natur. (8) 35 (1963), 244-251.

[2] A. Ben-Israel and T.N.E. Greville. Generalized inverses: Theory and Applications, 2nd Edition. Springer-Verlag, New York, 2003.

[3] J. Benítez, Moore-Penrose inverses and commuting elements of $C^{*}$-algebras, J. Math. Anal. Appl. 345 (2008), 766-770.

[4] C.Y. Deng, Reverse order law for the group inverses, J. Math. Anal. Appl. 382 (2011), 663-671.

[5] N.Č. Dinčić and D.S. Djordjević, Basic reverse order law and its equivalencies, Aequationes Math. 85 (2013), 505-517.

[6] D.S. Djordjević and V. Rakočević, Lectures on Generalized Inverses, Faculty of Science and Mathematics, University of Niš, Niš, 2008.

[7] M.P. Drazin, Pseudo-Inverses in Associative Rings and Semigroups, Amer. Math. Monthly, 65 (1958), 506-514.

[8] G.H. Golub and C.F. Van Loan, Matrix Computations, The John Hopkins University Press, Baltimore, MD, 1983.

[9] T.N.E. Greville, Note on the generalized inverse of a matrix product, SIAM Rev. 8 (1966), 518-512.

[10] S. Izumino, The product of operators with closed range and an extension of the reverse order law, Tohoku Math. J. 34 (1982), 43-52.

[11] J.J. Koliha, D.S. Djordjević, and D.S. Cvetković-Ilić, Moore-Penrose inverse in rings with involution, Linear Algebra Appl. 426 (2007), 371-381.

[12] C.D. Meyer; The role of the group generalized inverse in the theory of Markov chains; SIAM rev. 17 (1975), 443-464.

[13] D. Mosić and D.S. Djordjević, Reverse order law for the group inverse in rings, Appl. Math. Comput. 219 (2012), 2526-2534.

[14] D. Mosić and D.S. Djordjević, Some results on the reverse order law in rings with involution, Aequationes Math. 83 (2012), 271-282.

[15] K.P.S. Bhaskara Rao; The Theory of Generalized Inverses Over Commutative Rings; Taylor 
and Francis, 2002.

[16] C.R. Rao and S.K. Mitra, Generalized Inverse of Matrices and Its Applications, John Wiley, New York, 1971.

[17] H.J. Werner, G-Inverse of Matrix Products, Data Analysis and Statistical Inference, Eul-Verlag, Bergisch-Gladbach, 1992, 531-546.

[18] G. Wang, Y. Wei, and S. Qiao, Generalized Inverses: Theory and Computations, Science Press, Beijing, 2004.

[19] G. Wang and Z. Xu, The reverse order law for the $W$-weighted Drazin inverse of multiple matrices product; J. Appl. Math. Comput. 21, (2006) 239-248.

[20] J. Wang, H. Zhang, and G. Ji, A generalized reverse order law for the products of two operators, J. Shaanxi Norm. Univ. (4) 38 (2010), 13-17.

[21] F. Zhang; Matrix Theory, Basic Results and Techniques; 2nd Edition. Springer-Verlag, New York, 2011. 